IX.

\title{
Ein Fall von doppelseitiger Sinusthrombose mit einseitiger Jugularisunterbindung.
}

\author{
Von
}

Dr. Gust. Schlegel in Braunschweig.

(Mit 2 Temperaturkurven).

So viele Fälle von Sinusthrombose mit oder ohne Jugularisunterbindung auch schon in der Literatur niedergelegt sind, so finde ich doch in der mir zugängigen keine Beobachtung am L ebenden über doppelseitige Erkrankung des queren Hirnblutleiters. Daher scheint es mir angebracht, uber eine doppelseitige Sinusthrombose mit einseitiger Jngularisunterbindung, welche ich im letzten Winter zu beobachten Gelegenheit hatte, zu berichten.

Fräulein E. K. Kontoristin, 26 Jahre, kam am 20. August 05 in meine Behandlung mit akuter Mittelohrentzündung. Da dieselbe unter der üblichen Schwartze schen Behandlung nicht zurückging, wurde am 24. August parazentesiert. Die subkutiven Beschwerden verloren sich in den nächsten Tagen, die Eiterung nahm einen etwas protahierten aber normalen Verlauf. Ende September bestand noch geringe seröse Sekretion, das Gehör schon sehr gebessert. Nach einer in der ersten Oktoberhälfte zugezogenen Erkältung trat eine Verschlimmerung ein mit starken Erscheinungen von seiten des Warzenfortsatzes, welche trotz Antiphlogose und nachmaliger breit angelegter Parazentese nicht zurückgingen, so daß die AufmeiBelung nötig wurde.

19. Oktober Operation: Assistenzarzt Dr. Gravemann.

Schnitt in der üblichen Weise. Weichteile unverändert. Erweiterte Gefäßlöcher in der Fossa mastoidea. Nach wenigen Meißelschlägen quillt unter hohem Druck stehender Eiter hervor. Antrum mittelgroB, dasselbe ebenso wie die senkrechten Zellen voll frisch aussehender Granulationen. Die Diploe in großer Ausdehnung von Eiterpunkten durchsetzt, besonders nach hinten und unten. Die Spitze wird völlig reseziert, der Sinus transversus, dessen knöcherne Wandung ebenfalls erkrankt ist, in $11 / 2-2 \mathrm{~cm}$ Länge und in seiner ganzen Breite freigelegt, auch einige nach hinten rom Sinus, in der Hinterhauptschuppe gelegene Zellen werden eroffinet, auch diese sind mit gesch wollener dunkelroter Schleimhaut ausgekleidet. Sinuswand unverändert, leicht eindrückbar, pulsierend.

Die ersten acht Tage boten nichts Besonderes. Die Schmerzen verloren sich in zwei Tagen; das Allgemeinbefinden bob sich. nnd Pat. wurde am 25. Oktober zur ambulanten Behandlung aus der Klinik entlassen. Die Temperatur war während der klinischen Beobachtung nicht über 37,4 herausgekommen. 
25. Oktober. Verbandwechsel. Wunde gut aussehend, überall frisch granulierend. Tampon im Gehörgang nur an der Spitze durchfeuchtet, Trommelfell reizlos, Perforat. sehr verkleinert. Am 31. Oktober klagt Pat. wieder über Schmerzen in der linken Schläfe, nach dem Scheitel ausstrahlend, seit 2 Tagen auch wieder Nachlaß des Appetits, Obstipation, gestörtes Allgemeinbefinden, Temperatur nicht erhöht, Puls 70-80.

Beim Verbandwechsel níchts Besonderes nachweisbar, auch nach längerem Warten nirgends Eiterpunkt. Ohr trocken, Trommelfell geschlossen, Gehör für Flüstersprache $6-7 \mathrm{~m}$.

2. November. Zunabme der Schmerzen in der linken Scbläfe, so daß Pat. eine schlechte Nacht hatte. Temperatur abends 38, Puls 72. 78. Pat. hatte zu Hause täglich selbst gemessen, die höchste bisher beobachtete Temperatur war 37,3 gewesen. Schüttelfrost war nicht anfgetreten. Seit gestern liegt der Appetit ganz danieder.

Beim Verbandwechsel Druckempfindlichkeit über dem oberen Wundwinkel nach dem Jochbogen $z u$.

3. November. Status idem. In der Überzeugung, daß nach dem Jochbogen zu noch kranke Zellen zurúckgeblieben sind und mit der Möglichkeit eines extraduralen Abszesses der mittleren Schädelgrube rechnend, schlug ich der Pat. eine nochmalige Operation vor.

4. November. Nachoperation. Dr. Schlegel.

Verlängerung des Schnittes nach vorn bis über die Wurzel des Jochbogens. Es werden hier noch 2 größere und einige kleinere Zellen mit verdickter Schleimhaut eröffnet, Dura der mittleren Schädelgrube in 2 Markstäckgröße freigelegt, nirgends Eiter, Dura hyperămisch.

5. November $37,3.39$. geringer

Trotz der hohen Abendtemperatur Allgemeinbefinden besser, Schmerzen

6. November 37,8 . 38,4.

Schmerzen beute wieder erheblich stärker, am stärksten in der Schläfe. Allgemeinbefinden schlecht, Unlust, vollständige Appetitlosigkeit, geringer Fötor ex ore, Zunge stark belegt, trotz Karisbader Brustpulver kein Stublgang. Puls leicht unregelmäßig, 72-80.

7. November $37,5.39,4$.

Nacht trotz 0.02 Morph. sehr unruhig. Beim Verbandwechsel 4 malige Punktion des Schläfenlappens, ohne Eiter zu finden. Darauf Untersuchung der hinteren Schädelgrube, wobei die Verdickung der Sinuswand auffällt. Durch Probeinzision wird Thrombose festgestellt. In Narkose Aus. räumung des Sinus, welcher in seinem ganzen Verlaufe durch einen braunroten in der Mitte erweichten Thrombus ausgefullt ist. Nach Excision der ăußeren Wand Ausräumung mit dem scharfen Löffel peripheriwärts, bis starke Blutung erfolgt, zentralwärts bis nahe zum Bulbus, was leicht gelang, da bei der ersten Operation der Sinus durch breite Resektion weit freigelegt war. Eine Blutung trat hier nicht ein.

8. November $38,3.38,39,3$.

Schmerzen unverändert stark, jetzt aber mehr im ganzen Kopf, Allgemeine Unrube, Anorexie. Fötor ex ore stärker.

Im Laufe der linken Jugularis keine Beschwerden, nirgends Druckempfindlichkeit.

9 . November: $38,2, \quad 38,9$.

Auch diese Nacht trotz Morphium sehr unruhig. Status idem.

10. November: 37,4 . 38,3 .

11. November: $37,6.38$.

In den beiden letzten Tagen Besserung des Allgemeinbefindens. Die Schmerzen sind noch vorhanden, aber schwächer. Fine Änderung ist insofern eingetreten, als die Schmerzen aus der Schläfengegend verschwunden sind und jetzt mehr uber Nacken und Hinterkopf geklagt wird.

12. November $38,5.38,9$.

13. November: $38,9.39,5$.

14. November: 39,6 . 38,7 .
Befinden in diesen 3 Tagen wieder zunehmend schlechter. Appetit

Archiv f. Ohrenheilkunde. LXIX. Bd. 
liegt wieder ganz danieder, völlige Stuhlverhaltung. Intensive Kopfschmerzen im Hinterhaupt und Nacken, Nackenwirbel aber nicht druckempfindlich. Ophthalmoskopischer Befund normal.

14. November Punktion des Kleinhirns, negativ.

15.-20. November: Temperatur zwischen $37,5-39,1$.

Allgemeinbetinden etwas besser, da der Appetit wiederkehrt. Die Schmerzen halten dagegen mit kurzen Remissionen an, werden jetzt aber am meisten auf der rechte'n Seite, am stärksten am hinteren Rande des Warzenfortsatzes geklagt. Seit gestern leichter Druckschmerz in der Gegend des Emissarium mastoideum.

20.-23. November Temperatur zwischen $39,4-38,5$.

Die Schmerzen auf der rechten Seite unverändert. Druckschmerz am Foram. mastoideum stärker, kein Ödem. Warzenfortsatz selbst gar nicht empfindlich. Seit 2 Tagen Schmerzen im rechten Kieferwinkel, spontan and auf Druck. Die ptlegende Schwester hat gestern und heute mehrfach Rötung der rechten Gesichtshälfte beobachtet.

24. November: 38 . 39,2 .

Seit gestern abend Stechen beim Atmen in der rechten Brust. Seit 2 Tagen zeitweilig Hưsteln, heute Husten mit schleimigem Auswurf in geringer Menge.

Untersuchung der Lunge ergibt Dämpfung vorn oben rechts im 2. bis 3. Interkostalraum, Bronchialatmen mit einzelnen Rasselgeräuschen.

Ordin. Solut. Pyrenol 5/200. 3 mal täglich. Prießnitz.

Schmerzen hinter dem Warzenfortsatz und im Kieferwinkel heute weniger stark.

25. November: $38,2,39,2$, Status idem.

26. November: 38,3 . 38,9 ,

Husten mit Auswurf hente geringer, Sehmerzen beim Atmen verschwunden. Dämpfung in Aufhellung begriffen. Die Kopf- und Halsschmerzen waren in diesen beiden Tagen sehr viel besser gewesen, so dab mit Morphium, obne welches Pat. bisher nicht sein konnte, ausgesetzt worden war. Appetit trotz des Fiebers leidlich. und 38,5 .

27. November bis 2. Dezember: Temperatur schwankend zwischen 36,6

Befinden in diesen Tagen relativ gut. Schmerzen unbedeutend. Appetit und Stimmung gut. Seit 2 Tagen wieder spontaner Stuhlgang.

3. Dezember: $37,3,38,3$.

In letzter Nacht erneutes Einsetzen der rechtsseitigen Halsschmerzen, vom Kiefer bis zur Mitte des Halses heruntergehend. Die ganze seitliche Halsgegend ist bis uber den Larynx heraus bei der geringsten Berührung sebr schmerzhaft, bei stärkerem Druck, welcher die Pat, zu lautem Stöhnen bringt, ist die verdickte Ingularis deutlich za fühlen. Wegen der starken Schluckschmerzen wird Nahrungsaufnahme verweigert. Anwendung feuchter Wärme steigert die Schmerzen, Eisblase wird zuerst wobltuend, dann unangenehm empfunden. In 24 Stunden 3 mal 0.02 Morph. subkutan.

4. Dezember: $37,2.39,2$.

Seit gestern abend wieder Schmerzen in der rechten Brustseite. Heute Husten mit geringem, schleimigem Auswurf. Untersuchung ergibt Metastase im Mittellappen, Dämpfung und Bronchialatmen im V. Interkostalraum. Schmerzen trotz großer Morpbiumdosen unerträglich. Nahrungsaufnahme auch beute der Schmerzen wegen verweigert.

Ordinat. Solut. Pyrenol. $5 / 200$. Priebnitz.

5. Dezember: $37,6.39$.

Unterbindung der Jugularis: Dr. Schlegel.

Da die Verdickung der Jugularis noch unterhalb des Keblkopfes zu fühlen ist, wird der Schnitt in der Höhe des Schildknorpels beginnend bis zur Supraklavikulargrube geführt. Die Jugularis wird in einer Länge von zirka $4 \mathrm{~cm}$ freigelegt. Sie ist thrombosiert. Die Vene wird doppelt unterbunden oben dicht über der Cartilago cricoidea, unten $2 \mathrm{~cm}$ über der Clavicula. Bei der Spaltung zeigt sich die Wandung um das 4-5fache verdickt, das enge Lumen ausgefüllt durch einen festen braunroten Thrombus. 
Ein Fall v. doppelseit. Sinustbrombose m. einseit. Jugularisunterbind. 179
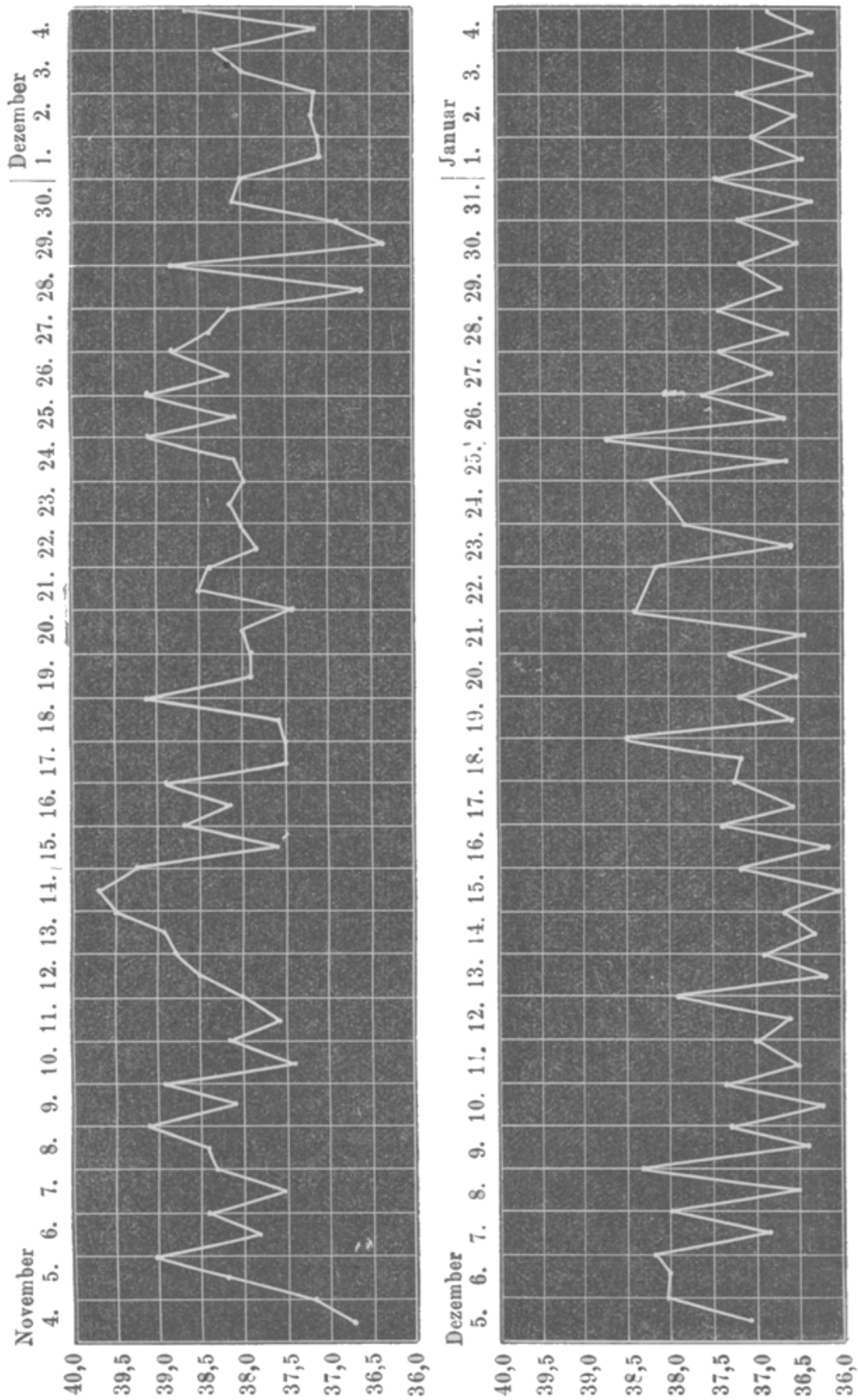
Das zwischen den Ligaturen gelegene Stäck der Vene wird excidiert, die Wunde bis auf eine kleine Partie unten zum Herausleiten der Ligaturen durch Nähte geschlossen.

6. Dezember: $36,5,38,2$.

Nacht trotz 0.03 morph. sehr unruhig. Viel Erbrechen. Pat. sehr an gegriffen, quälender Husten, Brustschmerzen unvorändert, ebenso Halsschmerzen, so daß Schlucken noch unmöglich.

7. Dezember: $36,9.38$.

Status idem, aber kein Erbrechen mehr. Heute fällt zum ersten Male leichte Heiserkeit der Patientin auf.

8. Dezember: $36,5,39,4$.

Brustschmerzen geringer, Husten nicht mehr so quälend, heute mehr Auswurf als gestern, leicht rötlich gefärbt. Zunabme von flüssiger Nabrung. Schluckschmerzen gemildert.

9. Dezember: $36,4,37,4$.

Leidliche Nacht nach Morph. 0.01 mit Trionol 0.5. Appetit kehrt zurück. Schluckschmerzen nur noch unbedeutend. Stimmung gut.

Beim Verbandwechsel nichts Besonderes. Halsgegend auf Druck nur noch wenig empfindlich.

10.-14. Dezember: Temperatur schwankend zwischen 36 und 37,9 .

Erscheinungen von seiten der Lunge zurückgebildet. Zeitweilig noch Schmerzen in der Mitte des Hinterkopfes und hinter dem Warzenfortsatz; dieselben sind aber erträglich und nur von kurzer Dauer. Appetit und Allgemeinbefinden gut.

11. Dezember: Entfernung der Nähte.

Vom 13. Pyrenol ausgesetzt, vom 12. kein Morphium mehr, abends 1.0 Trionol.

16. Dezember: Appetit jetzt sehr gut. Seit gestern wieder m ehr Schmerzen, von der Protuberantia occipitalis zum Scheitel heraufziehend. Für die Nacht wieder Morphium 0.02 .

18. Dezember: $37,2.35,5$.

Gestern und heute wieder starke Schmerzen, besonders nachts, trotz Morphiums keine Ruhe. Im rechten Ohr Schmerzen und zeitweilig Geräusche. Otoskopisch normal.

19.-20. Dezember: Temperatur wieder normal. Schmerzen besser, Appetit wieder besser. Beim Verbandwechsel Retention im unteren Wundwinkel.

22. Dezember: $35,4,38,2$.

Schmerzen in der letzten Nacht wieder unerträglich in der Mitte des Hinterkopfes, hinter dem Warzenfortsatz nach der Stirn und in das rechte Auge ausstrahlend. Eisblase auf die rechte Seite 3 mal $0.02 \mathrm{Morph}$. im Laufe des Tages. Aus dem unteren Wundwinkel der Unterbindungswunde reichliche Sekretion aus einem Senkungsabszeß. Erweiterung mit der Kornzange. Jugularisgegend nirgends mehr auf Druck empfindlich.

Von jetzt ab täglicher Verbandwechsel, Augenhintergrund normal.

23. -25. Dezember: $36,6-38,8$.

In diesen drei Tagen wieder unerträgliche Schmerzen in der ganzen rechten Kopfhälfte, besonders aber der Stirn, trotz Eisblase und großer Morphiumdosen. Schmerzen im rechten Ohr. Otoskopisch und ophthalmoskopisch nichts nachweisbar. Seit 2 Tagen wieder Nachlassen des Appetits.

26. Dezember: Status idem. Da Morphium nicht mehr vertragen wird, mehrmals tăglich 0.005 Heroin vom 24. $a b$.

27. Dezember: Nach besserer Nacht nur geringe Schmerzen, keine Injektion. Appetit und Stimmung wieder gut. Abends Trionol.

29. Dezember: Wieder mehr Schmerzen, uber dem rechten Auge und in der Schläfe. Ophthalmskopisch stärkere Fallung der venösen Gefaße?

30. Dezember: Befinden hente sehr gut. Appetit hervorragend. Pat. klagt über Scbwerhörigkeit rechts.

Otoskopisch normal.

Hörprüfung; Flüstersprache $1 / 4 \mathrm{~m}$. Weber nach links, Uhr vom Warzenfortsatz nach links, ebenso $C$ nach links. $C_{2}$ nur bei starkem Anschlag. 
Ein Fall v. doppelseit. Sinusthrombase m. einseit. Jugularisunterbind. 181

31. Dezember bis 4. Januar 06: Gutes Allgemeinbefinden. Zeitweilig noch mäfige Stirnkopfschmerzen.

6. Januar: Pat. steht zum ersten Male auf. Nach dem Aufsein wieder stärkere Schmerzen in der rechten Kopfhälfte.

Ophthalmoskopisch: Rechts Stauungspapille, links normal.

Gehör für Flüstersprache rechts wieder $21 / 2-3 \mathrm{~m}$.

Stimme immer noch leicht heiser. Larynogoskopische Untersuchung zeigt rechtsseitige Rekurrensparese.

13. Januar: Pat. ist jeden Tag etwas länger aufgestanden. Allgemeinbefinden und Appetit sehr gut. Pat. klagt seit gestern über schlechtes Sehen und Doppelbilder.

Ophthalmoskopisch beiderseits Staungspapille.

Rechtes $\mathrm{Ohr}$ wieder normal.

15. Januar: Untersuchung durch H. Dr. Hofimann, Augenarzt ergibt Bestätigung der Diagnose Stauungspapille mit mäßiger Herabsetzung des Visus und Doppelbildern, deren Natur sich nicht genau feststellen jäßt, welche aber auf Parese des Rectus externus und der beiden Obliqui zu bezieben sind.

Dje Rekonvaleszenz nahm nun einen ungestörten Verlauf. Die Augenbeschwerden nahmen in den näcbsten acht Tagen noch za, verloren sich dann aber im Laufe der nächsten drei Wochen. Der ophthalmoskopische Befund war erst Ende Februar wieder normal. Am 20. Januar wurde Pat. aus der Klinik entlassen.

Die Heilang der Empyemoperationswande, welche allerdings sehr tief und breit war, 'ging sehr langsam vor sich. Erst Ende März war dieselbe vernarbt, das Gehör auf dem linken $O$ br war schon vierzehn Tage nach der ersten Operation normal.

Auch die Unterbindungswunde, welche aus dem SenkungsabszeB reichlich sezernierte, war erst Mitte März verheilt.

Die Recurrensparese ist gebliében; irritiert aber die Patientin nicht, da die Sprache auch vorher immer etwas belegt gewesen ist.

\section{Epikrise.}

Bis zur ersten Operation bot der Fall nichts Besonderes. Als abnorm waren aber die acht Tage nach der Operation einsetzenden. Sehmerzen anzusprechen. Die ständige Verlegung derselben in die Schläfengegend verleitete zu der Annahme eines extraduralen Abszesses in der mittleren Schädelgrube. Die Nachoperation ergab zwar einige kranke Zellen, aber keinen extraduralen AbszeB. Da ein ursächlicher Zusammenhang zwischen den Sehmerzen und den noch vorhanden gewesenen kranken Zellen nicht ausgesehlossen war, ließ ich es bei der einfachen Nachoperation bewenden. Aber schon nach wenigen Tagen mußte ich die Überzengung gewinnen, daß den Beschwerden eine andere Ursache zugrunde liegen mußte. Die Erseheinungen - starke Schläfenkopfschmerzen, Unlust, Foetor ex ore, Anorexie und Obstipation - reohtfertigten die Annahme eines Schläfenlappenabszesses. Gegen Abszeb sprach allerdings die Temperatur, dieselbe konnte aber auch noch als protahiertes Resorptionsfieber aufgefaßt werden. An eine Erkrankung des Sinus dachte ich nicht, da nach Angabe der Patientin weder 
vorher noch während der klinischen Beobachtungszeit ein Schuttelfrost aufgetreten war. Das negative Ergebnis der Punktion des Schläfenlappens lieb mich mein Augenmerk auf die hintere Schädelgrube lenken, und nun wurde die Thrombose des Sinus transversus konstatiert. Derselbe wurde ausgeräumt, von einer Unterbindung der Jugularis aber Abstand genommen, da dieselbe zurzeit anscheinend gesund war und auch keine Metasta sen aufgetreten waren.

Ein ausgesprochener Einflu 3 der Operation war nicht erkennbar. Eine geringe Besserung des Allgemeinbefin dens trat zwar ein, aber nur sehr vorübergehend. Vom 12. an wn rde das Befinden erheblich schlechter, und da das Fieber ein en mehr kontinuierlichen Charakter annahm, glaubte ich eine Kompliplikation mit Meningitis: Hirnabszeß annehmen zu mü ssen und punktierte am 14. das Kleinhirn, aber auch ohne Erfolg.

Das Übergreifen der Schmerzen auf die rechte Hinterhauptseite in den Tagen vom 16. an erweckte zuerst den Verdacht auf ein Fortschreiten der Thrombose dureh den Confluens sinuum auf den rechten Sinus transversus, was nicht mehr zweifelhaft war, als sich vom 20. an ein ausgesprochener Druck schmerz am hinteren Rande des rechten Warzenfortsatzes einstellte mit gleichzeitig auftretenden Sohmerzen im Kieferwinkel. $\mathrm{Zu}$ einer Eröffnung des rechten Sinus konnte ich mich bei dem $z$ iemlich desolaten Zustand der Patientin nicht entschließen, um so weniger, als an eine gründliche Ausräumung der Thromb ose doch nicht zu denken war. Ein neues Moment kam in das Krankheitsbild mit dem Auftreten der Lungenmetastase. Es unterlag wohl keinem Zweifel, daß die Metastase von dem rechten Sinus ausgehen mußte; denn ein Zusammenhang mit der linken Thrombose nach so langer Zeit - 17 Tage - war um so unwahrseheinlicher, als die linke Jugularis frei geblieben war. In Frage kam jetzt die rechtsseitige Jugularisunterbindung, die in Vorschlag gebracht, von der Patientin aber abgelehnt wurde.

Nach Ablauf der Lungenmetastase folgten einige Tage relativen Wohlbefindens, bis am 2. Dezember die Schmerzen in der Kiefer- und seitlichen Halsgegend mit solcher Heftigkeit einsetzten, daß jede Schluckbewegung zur Qual und deshalb jede Nahrungsaufnahme verweigert wurde. Drängten nun diese Schmerzen sehon zur Jugularisunterbindung, so wurde sie zur absoluten Notwendigkeit, als am 4. eine neue Lungenmetastase einsetzte. Patientin, durch die anhaltenden Schluckschmerzen 
Ein F all v. doppelseit. Sinusthrombose m. einseit. Jugularisunterbind. 183

mürbe geworden, war mit der Unterbindung einverstanden, welche ich am 5. ausführte.

Der weitere Verlanf bot bis zum 17. nichts Besonderes. Am 18. trat nach neuntägiger Pause zum ersten Male wieder ein höherer Anstieg ein, nachdem in den letzten beiden Tagen viel Kopfschmerzen in der Mitte des Hinterkopfes von der Protuberantia bis zum Seheitel herauf bestanden hatten. Eine Erklärung für diese Schmerzen möchte ich in einer Mitbeteiligung des Sinus longitudinalis superior suchen. $\mathrm{Ob}$ diese Annahme richtig, ist sehwer zu sagen, da wir für die Diagnose einer partiellen Thrombose des Sinus longitudinalis noch keine sicheren Kennzeichen besitzen. Denn das von Gradenigo angegebene Symptom Scheitelschwellung - setzt schon eine ziemliche Ausdehnung der Thrombose voraus. Jedenfalls war diese Schwellung in unserem Falle nicht vorhanden, ebenso wenig die von Lermoyez beobachtete Erweiterung der Venen der behaarten Kopfhaut. Nicht ohne Bedeutung für die Wahrscheinlichkeitsdiagnose scheint mir der Umstand, daß die Thrombose des Sinus transversus eine rechtsseitige war wie in allen bisher sicher, d. h. durch Autopsie, festgestellten Fällen von Longitudinalisthrombose.

Diese Schmerzen in der Mitte des Hinterhauptes ließen nach drei Tagen nach, um nach einem kurzen freien Intervall wieder von auf der rechten Seite einsetzenden Schmerzen abgelöst zu werden; die Sehmerzen sitzen jetzt aber wieder in der Stirn und "hinter dem rechten Auge". Ich erwartete von einem Tag zum andern die Erscheinungen einer Cavernosus-Thrombose auftreten zu sehen. Zum Glück wurde diese Befürehtung nicht zur Tatsache. $\mathrm{Ob}$ diese Stirnkopfschmerzen als Folge der allgemeinen Stauung aufzufassen sind, oder ob vielleicht doch eine Thrombose des Sinus petrosus superior mit teilweiser Beteiligung des Cavernosus bestanden hat, lasse ich dahingestellt. Für eine Erkrankung des Sinus petrosus superior wirden die zu gleicher Zeit auftretenden Störungen des rechten Ohres zu verwerten sein. Nachdem Patientin schon mehrere Tage über zeitweilige Ohrschmerzen und Geräusche geklagt, machte sie am 30. zum ersten Male auf in den letzten Tagen aufgetretene Schwerhörigkeit aufmerksam, und die am selben Tage vorgenommene Prüfung ließ ein deutliches Ergriffensein des inneren Obres erkennen. Zu erklären ist diese Labyrinthaffektion wohl durch eine starke Hyperämie, analog der sich kurze Zeit darauf manifestierenden Staungspapille. Die Ausfallserscheinungen des rechten Ohres 
verloren sich in kurzer Zeit wieder, so daß nach 14 Tagen die Prüfung wieder normale Verhältnisse ergab. Diese Labyrinthhyperämie kann allerdings auch durch die Transversusthrombose allein bedingt sein, wird aber doch wobl meistens auf Staungsverhältnisse im Sinus petrosus superior bezogen.

Bei der ausgedehnten Ausschaltung des venösen Rückflusses war zu erwarten, daß sich ziemlich frubzeitig Veränderungen am Augenhintergrund zeigen mußten. Nachdem schon im November und erste Hälfte Dezember einige Male ohne Erfolg ophthalmoskopiert worden war, untersuchte ich vom 22. Dezember, an welehem Tage zuerst ibber starke Schmerzen "hinter dem Auge" geklagt wurde, regelmäßig in zweitägigen Pausen. Aber erst am 7. Januar war eine stärkere Fullung der Venen nachweisbar, welche in den nächsten acht Tagen zu einer regelrechten Stauungspapille sich ausbildete. Auffällig ist, daß der Patientin der Nachlaß des Sehvermögens und die Doppelbilder erst so spät zum Bewußtsein kamen. Entsprechend dem langsamen Entstehen der Staungspapille ging auch die Rlickbildung viel langsamer vor sich, als bei der Ohraffektion, erst Ende Februar war der Augenspiegelbefund wieder normal.

Es bliebe nooh zu erörtern die Entstehung der Recurrensparese, der Zeitpunkt des Einsetzens der Thrombose und der Ausgang ohne Unterbindung.

Auf welche Weise die Recurrensparese entstanden ist, hat sich nicht nachweisen lassen. Daß eine Verletzung des Vagus selbst oder wenigstens des Laryngeus inferior erfolgt sein muf, ist selbstverständlich, da die Heiserkeit gleich am Tage nach der Unterbindung bemerkt wurde. Über Recurrensparese nach Jugularisunterbindung, finde ich in der mir zugängigen Literatur nur eine kurze Notiz von Jansen, welcher in der Besprechung der Jugularisunterbindung in der Encyklopädie eine Recurrensparese erwähnt. Eine Verletzung des Recurrens würde, wenn die Unterbindung so weit unten angelegt wird, dann leichter eintreten können, wenn der Laryngeus inferior direkt vom Stamm zum Vagus tritt, ohne den Umweg um die Subclavia zu machen. $\mathrm{Ob}$ dieser zuweilen vorkommende abnorme Verlauf des Laryngeus inferior in unserem Falle bestanden hat, kann ich nicht sagen.

Mit größerer Wahrscheinlichkeit läßt sich ein Urteil abgeben uber die Zeit der Entstehung der Thrombose. Zweifellos war der Sinus zur Zeit der ersten Operation makroskopisch noch ge- 
Ein Fall v. doppelseit. Sinusthrombose $\mathrm{m}$. einseit. Jugularisunterbind. 185

und; ob nicht schon damals aber ein kleiner randständiger Thrombus vorhanden gewesen, läbt sich natürlich nicht aussohließen. Wahrseheinlieher jedoeh haben wir die Entstehung in die Zeit vom 28. an zu verlegen, da von da an wieder Schmerzen und aueh Störungen im Allgemeinbefinden auftraten. Auffällig ist, daß, wie schon oben bemerkt, während der ganzen Zeit nicht ein typischer Schlittelfrost - den Aussagen der intelligenten Patientin ist zu glauben - ja bis zum 1. November kein Fieber aufgetreten ist.

Über den Ausgang der Erkrankung ohne Unterbindung der Jugularis kann Zweifel sein. Es ist wohl unter Berlicksichtigung der zweimaligen Lungenmetastase und des Fortschreitens der Jugularisthrombose mit Sicherheit anzunehmen, daß die Erkrankung ohne Unterbindung der Jugularis zum Tode gefthhrt haben würde. Andererseits hätte sich möglicherweise das Übergehen der Thrombose auf die rechte Seite mit allen ihren Folgen verhüten lassen, wenn bei der Ausräumung des linken Sinus transversus auch die zweifellos damals schon bestehende Bulbusthrombose mit ausgeräumt worden wäre. So wïrde auch unser Fall wieder eine Mahnung sein, die immer mehr in Aufnahme kommende Methode, bei Sinusthrombosen den Bulbus jugularis mit auszuräumen, zu befolgen, da nach den Sektionsbefunden eine Fortleitung der Thrombose auf die ohrgesunde Seite doch nicht so selten ist, als man nach der klinischen Beobachtung erwarten sollte. 